OPEN ACCESS

Edited by:

Katsumi Maenaka,

Hokkaido University, Japan

Reviewed by:

Takashi Saitoh

Hokkaido College of Pharmacy, Japan

Toyoyuki Ose,

Hokkaido University, Japan

${ }^{*}$ Correspondence:

Hongying Chen

chenhy@nwafu.edu.cn

Specialty section:

This article was submitted to

Virology,

a section of the journal

Frontiers in Microbiology

Received: 12 March 2017

Accepted: 26 April 2017

Published: 11 May 2017

Citation:

Chen J, Xu X, Tao H, Li Y, Nan H,

Wang Y, Tian M and Chen $H$ (2017)

Structural Analysis of Porcine

Reproductive and Respiratory

Syndrome Virus Non-structural

Protein $7 \alpha$ (NSP7 $\alpha$ ) and Identification

of Its Interaction with NSP9.

Front. Microbiol. 8:853.

doi: 10.3389/fmicb.2017.00853

\section{Structural Analysis of Porcine Reproductive and Respiratory Syndrome Virus Non-structural Protein $7 \alpha$ (NSP7 $\alpha)$ and Identification of Its Interaction with NSP9}

\author{
Jiaping Chen ${ }^{1}$, Xiaodong Xu', Hu Tao ${ }^{2}$, Yuan Li', Hao Nan', Yuanyuan Wang', \\ Mengmeng Tian ${ }^{1}$ and Hongying Chen ${ }^{1 *}$ \\ ${ }^{1}$ College of Life Sciences, Northwest A\&F University, Yangling, China, ${ }^{2}$ College of Science, Northwest A\&F University, \\ Yangling, China
}

Non-structural protein 7 (NSP7), which can be further cleaved into NSP7 $\alpha$ and NSP7 $\beta$, is one of the most conserved proteins of porcine reproductive and respiratory syndrome virus (PRRSV). NSP7 plays a role in provoking the humoral immune system in PRRSVinfected swine, but its structure and function are still not fully understood. Here, we analyzed the expression of NSP7, NSP7 $\alpha$, and NSP7 $\beta$ in PRRSV-infected MARC-145 cells. The solution structure of NSP7 $\alpha$ was determined by using nuclear magnetic resonance (NMR). Although the structure provided little clue to its function, based on the structure of NSP7 $\alpha$, we predicted and further identified some key amino acids on NSP7 $\alpha$ for the interaction of NSP7 $\alpha$ with NSP9, the RNA dependent RNA polymerase of PRRSV. This study provided some new insights into the structure and function of PRRSV NSP7.

Keywords: porcine reproductive and respiratory syndrome virus, non-structural protein $7 \alpha$, protein structure, NMR, protein-protein interaction

\section{INTRODUCTION}

Porcine reproductive and respiratory syndrome virus (PRRSV) is an enveloped virus containing a positive single-stranded RNA genome of $15.4 \mathrm{~kb}$. Together with lactate dehydrogenase elevating virus (LDV), equine arteritis virus (EAV), simian hemorrhagic fever virus (SHFV), and wobbly possum disease virus (WPDV), they form the family Arteriviridae (Plagemann and Moennig, 1992; Snijder and Meulenbdrg, 1998; Dunowska et al., 2012). PRRSV is the causative agent of PRRS, which is one of the most destructive disease in swine industry in the world. Based on genetic differences, PRRSV strains are divided into European genotype and North American genotype and the nucleotide identity between the two genotypes is only about 60\% (Allende et al., 1999; Nelsen et al., 1999; Forsberg, 2005).

The genome of PRRSV contains at least nine open reading frames. Of these, ORF1a and ORF1b occupy $75 \%$ of the genome and encode polyproteins ppla and pplab. Thereafter, the two polyproteins could be cleaved into non-structural proteins (NSP1-12) by proteases NSP $1 \alpha, N S P 1 \beta$, NSP2, and NSP4 (van Aken et al., 2006; Fang and Snijder, 2010; Li et al., 2015). 
Among these NSPs, the roles of NSP5, NSP6, NSP7, and NSP12 in virus biology are still not understood.

ELISA analysis for NSP1, NSP2, NSP4, NSP7, and NSP8 showed NSP1, NSP2, and NSP7 could strongly react with pig serum, and the NSP7 dual ELISA can be used as a differential test for PRRSV serology (Brown et al., 2009). Previous research indicated that NSP3-8 might play a main role in PRRSV virulence (Kwon et al., 2008). A recent report showed that deletions in NSP7 led to failures in the recovery of PRRS virus (Zhang et al., 2013). These data suggest that the protein plays an important role in the life cycle of PRRSV.

With an internal cleavage site, NSP7 can be further cleaved into NSP7 $\alpha$ and NSP7 $\beta$ (Li et al., 2012). The cleavage site located within NSP7 is conserved in arteriviruses and critical for the replication of EAV (van Aken et al., 2006). Nuclear magnetic resonance (NMR) assay of the structure of EAV NSP7 $\alpha$ showed that the protein has three $\alpha$-helices and five $\beta$-strands. Although the structure analysis possesses no recognizable functional motifs, EAV NSP7/NSP7 $\alpha$ was proved to play an important role in viral RNA synthesis by structure-based reverse genetics studies (Manolaridis et al., 2011), consisting with the data from PRRSV (Zhang et al., 2013).

In this study, we analyzed the expression of NSP7 $\alpha$ and NSP7 $\beta$ in PRRSV infected cells and determined the structure of NSP7 $\alpha$ by NMR. Furthermore, we demonstrated the interaction of NSP7 $\alpha$ with NSP9, the viral RNA dependent RNA polymerase (RdRp), and identified the key amino acids of NSP7 $\alpha$ involved in NSP7 $\alpha-$ NSP9 interaction by mutagenesis analysis.

\section{MATERIALS AND METHODS}

\section{Cells, Viral Strains}

MARC-145 cells were maintained in Dulbecco's modified Eagle's medium (HyClone) supplemented with $10 \%$ fetal bovine serum (HyClone) at $37^{\circ} \mathrm{C}$ with $5 \% \mathrm{CO}_{2}$. TA-12 (GenBank accession no. HQ416720.1), a highly pathogenic PRRSV isolate, was used in virus infection studies.

\section{Bioinformatics Analysis}

The web tool of PDBeMotif ${ }^{1}$ was used for analyzing the motifs which may be functional in NSP7 $\alpha$. The alignment of the protein sequences were completed using DNAMAN software. PRISM2.0 2 was used for the prediction of bonding sites between NSP7 $\alpha$ and NSP9 (Tuncbag et al., 2011).

\section{Plasmid Constructs for the Expression of HIS-TEV-NSP $7 \alpha / \beta$}

The full-length PRRSV cDNA clone FL12 of NVSL 97-7895 (GenBank accession no. AY545985.1) was used as the templates for the amplification of the cDNAs encoding NSP7 $\alpha$ and NSP7 $\beta$. The fragment of $n s p 7 \alpha$ or $n s p 7 \beta$ was then inserted into a vector as described in a previous study (Chen et al., 2016), for the

${ }^{1}$ http://www.ebi.ac.uk/pdbe-site/pdbemotif/

${ }^{2} \mathrm{http}: / /$ cosbi.ku.edu.tr/prism/ expression of NSP7 $\alpha$ or NSP7 $\beta$ with an N-terminal HIS-tag and a TEV protease cleavage site between the tag and the fused protein.

\section{Polyclonal Antibodies against NSP7 $\alpha$ and NSP7 $\beta$}

HIS-TEV-NSP7 $\alpha$ and HIS-TEV-NSP7 $\beta$ were expressed in E. coli BL21 (DE3), and purified using Ni-NTA column (CWBIO, China). The $200 \mu \mathrm{g}$ of each purified protein was mixed with equal volume of Freund's complete adjuvant, and injected into rabbits in primary immunization. Rabbits were boosted three times at 2 weeks intervals, with mixture of $200 \mu \mathrm{g}$ antigen in Freund's incomplete adjuvant. The rabbit antiserums were collected 2 weeks after the last injection.

\section{Western Blot Assay}

MARC-145 cells were infected with a MOI of 0.1 , and lysed in SDS loading buffer at 8, 16, 24, 48, 72, and $96 \mathrm{~h}$ post infection (hpi). The proteins in cell lysis solution were separated by SDS-PAGE, and transferred onto a PVDF membrane. After blocking with $5 \%$ skimmed milk overnight at $4^{\circ} \mathrm{C}$, the membranes were, respectively, incubated with anti-NSP7 $\alpha$ and anti-NSP7 $\beta$ polyclonal antibodies, followed by incubation with goat-anti-rabbit IgG (CWBIO, China) conjugated horseradish peroxidase as secondary antibody. Immunodetection was performed using enhanced chemiluminescence (ECL) reagents (CWBIO, China) according to the supplier's instruction.

\section{Immunofluorescence Assay}

Porcine reproductive and respiratory syndrome virus-infected MARC-145 cells were fixed at $8,16,24,48$, and 72 hpi using $4 \%$ paraformaldehyde, permeabilized using $0.2 \%$ Triton X-100 for $10 \mathrm{~min}$ at room temperature, then blocked with $5 \%$ skimmed milk overnight at $4^{\circ} \mathrm{C}$. Subsequently, the cells were, respectively, incubated with polyclonal antibodies against NSP7 $\alpha$ and NSP7 $\beta$ for $1 \mathrm{~h}$. After incubated with FITCconjugated goat-anti-rabbit IgG (CWBIO, China) for $1 \mathrm{~h}$, the cells were stained with PI for $5 \mathrm{~min}$. The fluorescence images were captured using a confocal microscope (Nikon, Japan).

\section{Preparation of the NSP7 $\alpha$ Solution for NMR Experiments}

Uniformly labeled NSP7 $\alpha$ was expressed in E. coli BL21 (DE3) grown in M9 minimal media containing $0.2 \%(\mathrm{w} / \mathrm{v}){ }^{13} \mathrm{C}$ Glucose and $0.1 \%(\mathrm{w} / \mathrm{v}){ }^{15} \mathrm{~N} \mathrm{NH}_{4} \mathrm{Cl}$ as the only carbon and nitrogen sources. HIS-tagged NSP7 $\alpha$ was purified by affinity chromatography on a Ni-NTA column. The HIS-tag at the N terminus of NSP7 $\alpha$ was removed overnight by TEV protease, subsequently. The final NMR sample was concentrated to about $0.8 \mathrm{mM}$ in $20 \mathrm{mM} \mathrm{KH}_{2} \mathrm{PO}_{4}$ buffer ( $\mathrm{pH} 7.5$ ) with $92 \% \mathrm{H}_{2} \mathrm{O} / 8 \%$ $\mathrm{D}_{2} \mathrm{O}, 100 \mathrm{mM} \mathrm{NaCl}$, and $1 \mathrm{mM}$ TCEP. The residues of NSP7 $\alpha$ were renumbered as residues 2-150 (the glycine from the C-terminus of TEV protease cleavage site was numbered as residue 1) in structural analysis. 
TABLE 1 | The primers used in this study.

\begin{tabular}{|c|c|}
\hline Primers & Sequences \\
\hline EcoRINSP7 $\alpha$-F (yeast two-hybrid) & TAATGAATTCTCGCTGACTGGTGCCCTC \\
\hline BamHINSP7 $\alpha-R$ (yeast two-hybrid) & TAATGGATCCCTCCAGAACTITCGGTGG \\
\hline BamHINSP7 $\alpha-F$ (pull-down assay) & TAATGGATCCTCGCTGACTGGTGCCCTC \\
\hline XholNSP7 $\alpha-R$ (pull-down assay) & TAATCTCGAGTCACTCCAGAACTITCGGT \\
\hline NSP7 $\alpha$ K61A-F & TTGATGCGGTTCGAGGTACTITG \\
\hline NSP7 $\alpha$ K61A-R & TACCTCGAACCGCATCAACCTGCAC \\
\hline NSP7 $\alpha$ L69A-F & GGCCAAAGCTGAAGC ПITGCTGAT \\
\hline NSP7 $\alpha$ L69A-R & AAGCTTCAGCTाTGGCCAAAGTACC \\
\hline $\mathrm{NSP} 7 \alpha \mathrm{F} 72 \mathrm{~A}-\mathrm{F}$ & CTTGAAGCTGCTGCTGATACCGTG \\
\hline NSP7 $\alpha$ F72A-R & ATCAGCAGCAGCTTCAAGTITGGC \\
\hline
\end{tabular}

Restriction enzyme sites are underlined.

\section{NMR Experiments, Analysis of Spectra, and Structure Calculation}

NMR assignment of ${ }^{15} \mathrm{~N},{ }^{13} \mathrm{C}$ labeled $\mathrm{NSP} 7 \alpha$ was described in our previous report, and the ${ }^{1} \mathrm{H},{ }^{13} \mathrm{C}$, and ${ }^{15} \mathrm{~N}$ chemical shifts for NSP7a were deposited in the BMRB under BMRB accession number 30014 (Chen et al., 2016). All the NMR experiments were carried out at $298 \mathrm{~K}$ on a Bruker Avance $600 \mathrm{MHz}$ spectrometer. All spectra were processed using NMRPipe/Draw (Delaglio et al., 1995) and analyzed using CARA ${ }^{3}$ (Keller, 2004).

The structure of NSP7 $\alpha$ was refined by CNS (Brünger et al., 1998) based on the chemical shift assignments and NOE distance restraints. Backbone $\varphi$ and $\psi$ dihedral angle restraints obtained using TALOS+ (Shen et al., 2009). H-bond restraints were determined by secondary structure elements in initial structure. The final 20 structures with lowest energies were selected for representation and analyzed using PROCHECK_NMR (Laskowski et al., 1996). All representing structure figures were generated using PyMOL ${ }^{4}$.

\section{Yeast Two-Hybrid Analysis}

To investigate the interaction of NSP7 $\alpha$ with the other NSPs, yeast two-hybrid assays were performed. NSP7 $\alpha$ cDNA fragment of NVSL 97-7895 was cloned into pGADT7 and the other

${ }^{3}$ http://www.nmr.ch

${ }^{4}$ http://www.pymol.org/
NSPs' cDNA fragments were inserted into pGBKT7. Primer sequences for $n s p 7 \alpha$ cloning were listed in Table 1. The pGBKT7 constructs were, respectively, co-transformed with pGADT7NSP7 $\alpha$ into Saccharomyces cerevisiae (yeast) strain Gold using the Yeastmaker Yeast Transformation System kit (Clontech, USA). Yeast co-transformed with pGBK7-P53 and pGADT7-T was used as a positive interaction control, and yeast co-transformed with pGBKT7-Lam and pGADT7-T was used as a negative control.

\section{Pull-Down Assay}

The cDNA fragment encoding NSP7 $\alpha$ was amplified by PCR using primer pairs BamHINSP7 $\alpha-F / X h o I N S P 7 \alpha-R$. The fragment of $n s p 7 \alpha$ was inserted into pTriEx-FLAG for the expression of FLAG-tagged NSP7 $\alpha$. The site-directed mutants of $n s p 7 \alpha$ were amplified using primers listed on Table 1 and cloned into pTriEx-FLAG. NSP9 cDNA fragment was cloned into pTriEx1.1 vector to make pTriEx-NSP9 construct for the expression of HIS-tagged NSP9 (Liu et al., 2016).

Protein mixtures of FLAG-tagged NSP7 $\alpha$ and HIS-tagged NSP9 were incubated for $1 \mathrm{~h}$ at room temperature. The solution was incubated with nickel magnetic beads (Millipore, USA) for $0.5 \mathrm{~h}$ at room temperature. After being washed with wash buffer (20 mM TRIS-HCl [pH 7.9], $300 \mathrm{mM} \mathrm{NaCl}$, and $10 \mathrm{mM}$ imidazole) for three times, the bound proteins were eluted with elution buffer (20 mM TRIS-HCl [pH 7.9], $300 \mathrm{mM} \mathrm{NaCl}$, and $300 \mathrm{mM}$ imidazole). Eluted proteins were separated by SDSPAGE, and then detected by Western blot. FLAG-tagged and HIS-tagged proteins were, respectively, probed by mouse antiFLAG antibody (CWBIO, China) and mouse anti-HIS (CWBIO, China) antibody, followed by incubation with goat-anti-mouse IgG (CWBIO, China) conjugated with horseradish peroxidase as secondary antibody. The protein mixture containing NSP7 $\alpha$ and HIS-tagged TEV protease was used as negative control.

\section{RESULTS}

\section{Expression of NSP $7 \alpha / \beta$ in the PRRSV-Infected Cells}

Antisera against NSP7 $\alpha$ and NSP7 $\beta$ were used for the detection of NSP7 $\alpha$ and NSP7 $\beta$ expression in PRRSV infected MARC-145 cells. By Western blot, anti-NSP7 $\alpha$ antibodies detected a band
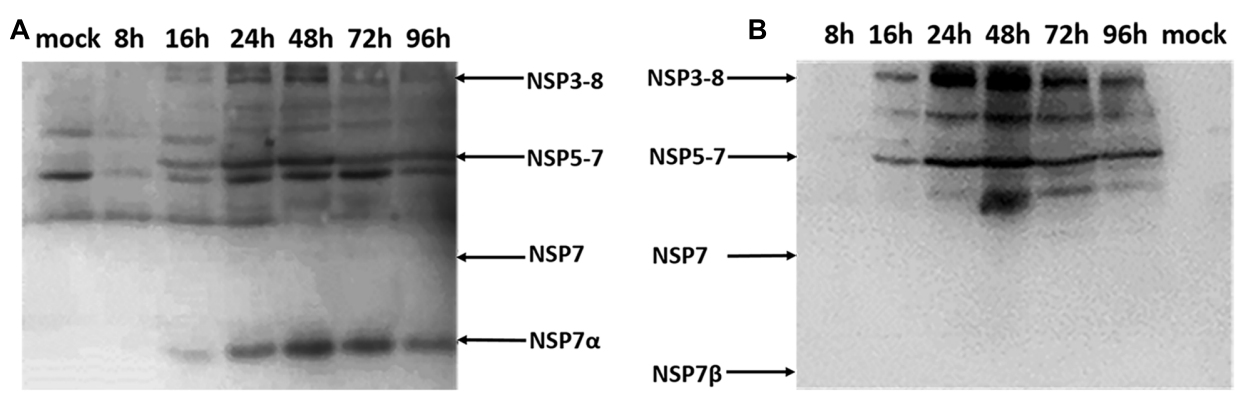

FIGURE 1 | Western blot analysis of the NSP7 expression in PRRSV-infected cells. Cell lysates were collected at 8, 16, 24, 48, 72, and 96 hpi, and probed with antisera against NSP7 $\alpha$ (A) and NSP7 $\beta$ (B). 


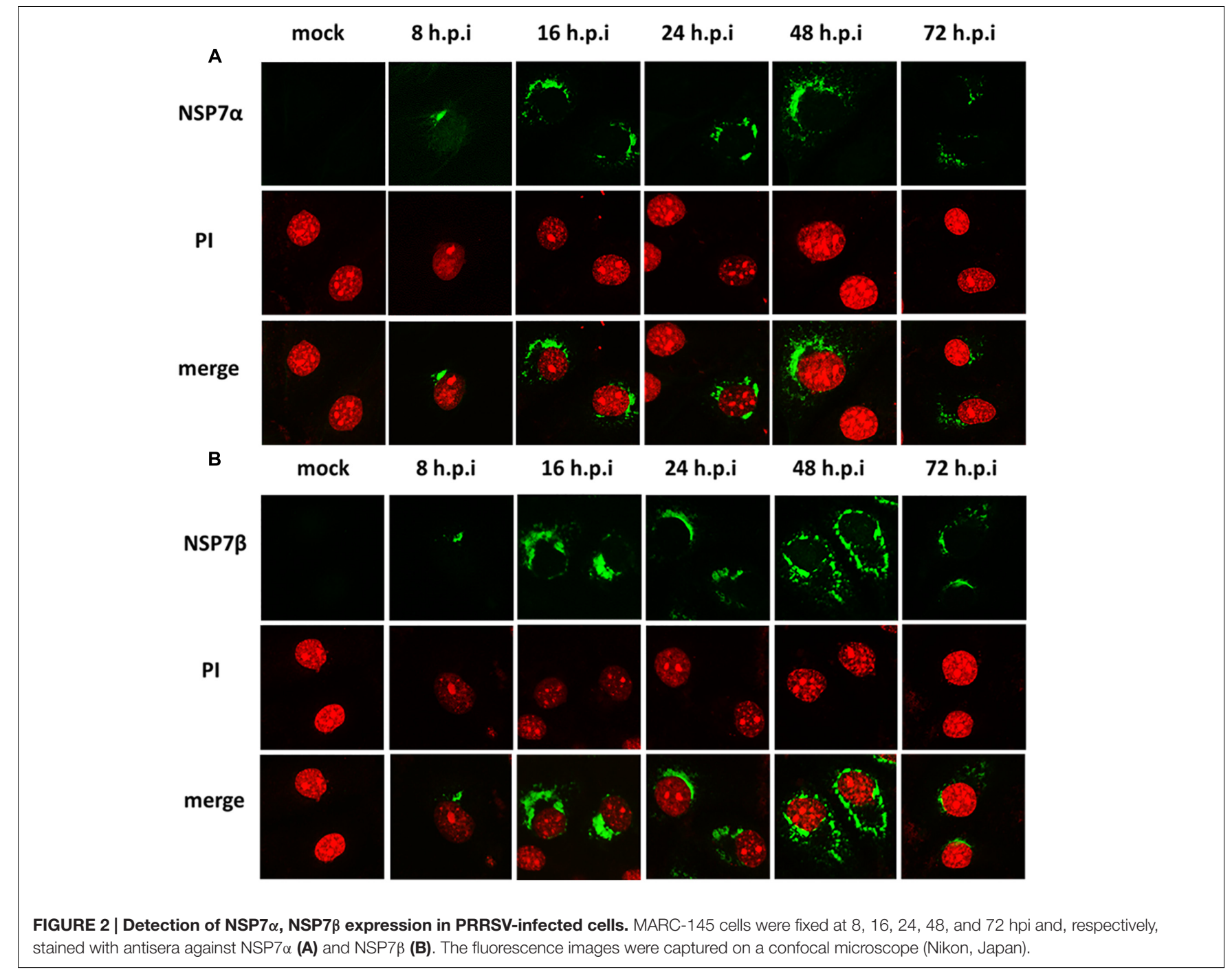

of approximately $16 \mathrm{kDa}$, corresponding to the predicted size of NSP7 $\alpha$, by 16 hpi (Figure 1A). The production of this protein reached a peak level at $48 \mathrm{hpi}$, and the protein amount gradually decreased from 48 to 96 hpi. A series of protein bands at higher molecular weights were also recognized. These bands probably represented the polyprotein precursors of NSP7, and the products in accordance with the predicted size of NSP3-8 and NSP5-7 were marked with arrows in the figure. However, no protein with the predicted size of NSP7 were detected in the PRRSV infected cells.

The antiserum against NSP7 $\beta$ also recognized the polyprotein precursors NSP3-8 and NSP5-7 (Figure 1B). It detected neither NSP7 nor NSP7 $\beta$ in the PRRSV infected cell lysates, probably due to the instability characteristics of the proteins. These results were consisted with the previous study that was carried out using PRRSV monoclonal antibodies (Li et al., 2012).

To visualize the intracellular expression and localization of NSP7 and its precursors, PRRSV infected MARC-145 cells were stained with the antisera and observed by immunofluorescence microscopy. Although the antiserum against NSP7 $\beta$ did not detect the cleaved form of NSP7 $\beta$ in Western blot, the two sera gave similar results in immunofluorescence assay, probably because the antiserum against NSP7 $\beta$ could detect the NSP7 precursors as well as the antiserum against NSP7 $\alpha$. As shown in Figure 2, the signal for NSP7 $\alpha$ and NSP7 $\beta$ could be detected in the infected cells as early as $8 \mathrm{hpi}$ and the fluorescent foci were small and mostly concentrated on one side of the nucleus. After 16 hpi, the fluorescence signals for both NSP7 $\alpha$ and NSP7 $\beta$ were more diffused in the cytoplasm of the infected cells. By $72 \mathrm{hpi}$, both the number of fluorescent cells and the intensity of the fluorescence obviously decreased. Protein degradation, and partially loss of the infected cells during the experimental process could both contribute to the reduction of the NSP proteins.

\section{Solution Structure of PRRSV NSP7 $\alpha$}

The solution structure of PRRSV NSP7 $\alpha$ was determined by NMR spectroscopy, and three dimensional structure was calculated using $2466 \mathrm{NOE}$ distance restraints (inter-residue), 202 dihedral angle restraints and $85 \mathrm{H}$-bond restraints. Coordinates of the ensemble of the 20 lowest energy structures 
TABLE 2 | Summary of structural statistics for NSP7 $\alpha$ structure.

\begin{tabular}{lc}
\hline Structural statistic & Value for NSP7 $\alpha$ \\
\hline Total distance restraints (inter-residue) & 2466 \\
Short range (residue $\mathrm{i}$ to $\mathrm{i}+\mathrm{j}, \mathrm{j}=1$ ) & 938 \\
Medium range (residue $\mathrm{i}$ to $\mathrm{i}+\mathrm{j}, 2 \leq \mathrm{j} \leq 4)$ & 672 \\
Long range (residue $\mathrm{i}$ to $\mathrm{i}+\mathrm{j}, \mathrm{j}>$ 4) & 856 \\
Hydrogen bonds & 85 \\
Total dihedral angle restraints & 202 \\
$\varphi$ & 101 \\
$\Psi$ & 101 \\
Restraint violations & \\
Distance restraint violation $0.2 \AA$ & 0 \\
Dihedral restraint violation $5^{\circ}$ & 0 \\
Average rmsd ( $\AA$ ) among the 20 refined structures & \\
Backbone of structured regions (residues 3-129) & $0.378 \pm 0.102$ \\
Heavy atoms of structured regions (residues 3-129) & $0.763 \pm 0.079$ \\
Ramachandran statistics of 20 structures (\% residues) & \\
Most favored regions & 63.73 \\
Additional allowed regions & 33.33 \\
Generously allowed regions & 1.67 \\
Disallowed regions & 1.27 \\
\hline
\end{tabular}

were deposited in the Protein Data Bank ${ }^{5}$ under PDB accession number 5I65. Parameters of the structure are listed in Table 2.

${ }^{5}$ http://www.rcsb.org/pdb/
As shown in Figure 3A, the ensemble of structures for the most domains are well superposed. NSP7 $\alpha$ contains a bundle of three $\alpha$-helices ( $\alpha 1$ from residue Asp13 to Lys21, $\alpha 2$ from residue Phe41 to $\mathrm{Gln} 56, \alpha 3$ from residue Gln59 to Ala74) and six-strand mixed $\beta$-sheet ( $\beta 1$ containing residues Cys27 to Val29, $\beta 2$ containing residues Ile86 to Leu90, $\beta 3$ containing residues Phe99 to Val103, $\beta 4$ containing residues His 108 to Glu114, $\beta 5$ containing residues Arg116 to Ala119 and $\beta 6$ containing residues Met123 to Val129 in the order $\alpha 1-\beta 1-\alpha 2-\alpha 3-\beta 2-\beta 3-\beta 4-\beta 5-\beta 6$. The mixed $\beta$-sheet is formed by five antiparallel strands ( $\beta 2$ to $\beta 6)$ and one short parallel strand ( $\beta 1$ ) (Figure 3B).

In order to search for the functional motifs of NSP7 $\alpha$, the protein structure was analyzed using web tools of PDBeMotif but no recognizable functional motifs was identified. The surface charge distribution of the protein shows that the protein has some scattered electropositive and electronegative patches, and more of the electropositive patches locate on the surface of the $\beta$-sheet (Figure 3C).

Sequence alignment shows that PRRSV (NVSL 97-7895) NSP7 $\alpha$ shares $33.33 \%$ identity with EAV (Bucyrus strain) NSP7 $\alpha$ (Figure 4). It is noteworthy that the C-terminus of PRRSV NSP7 $\alpha$ has a predominant proline-rich region (PRR), with 10 prolines out of the last 20 amino acids. In comparison, the C-terminus of EAV NSP7 $\alpha$ is shorter and lack the PRR, but its NSP7 $\beta$ N-terminus has four proline residues and five other same amino acids as the C-terminus of PRRSV NSP7 $\alpha$ and shares

${ }^{6}$ http://www.ebi.ac.uk/pdbe-site/pdbemotif/
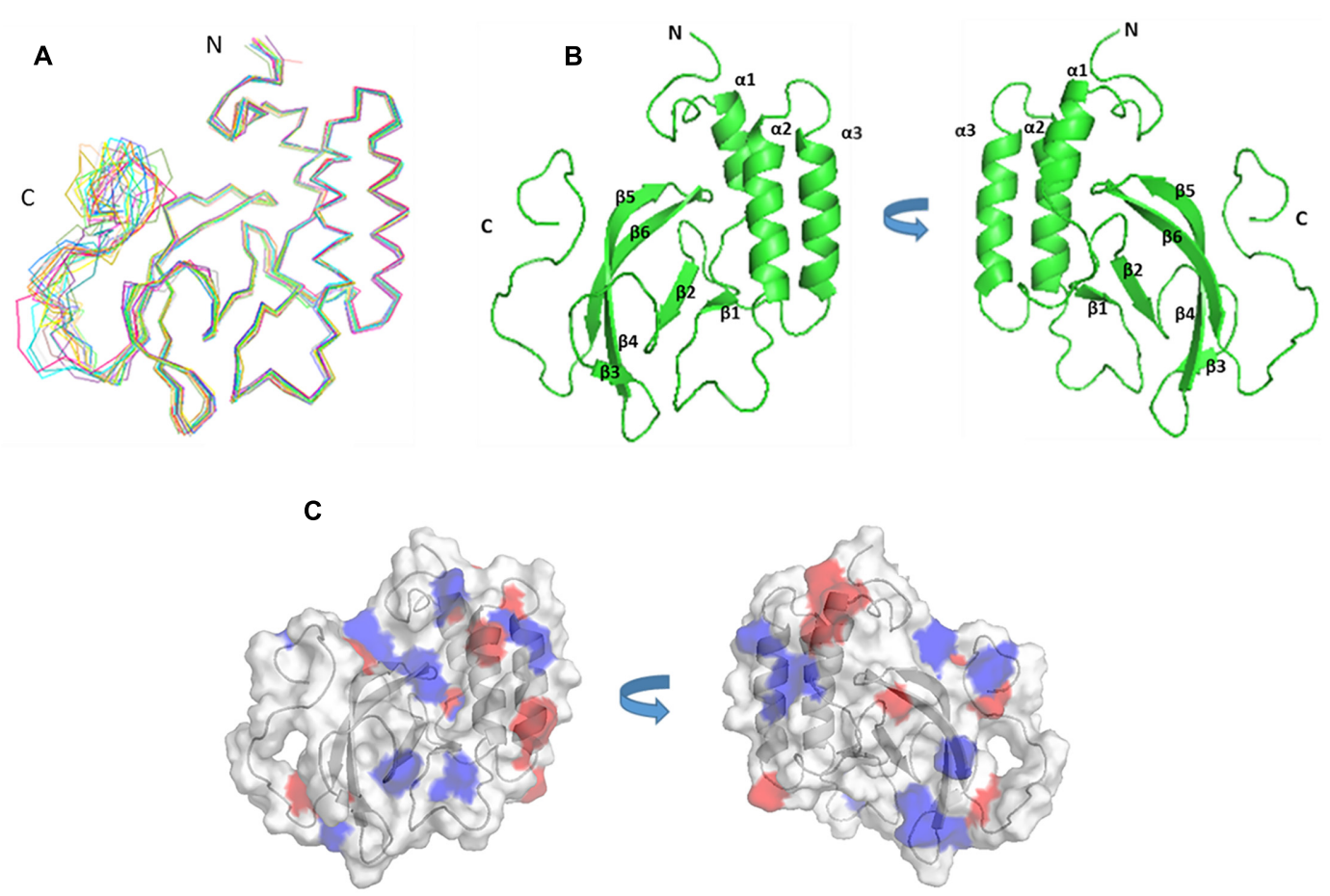

FIGURE 3 | The structure and surface features of PRRSV NSP7 $\alpha$ determined by NMR Spectroscopy. (A) Backbone trace of the ensemble of 20 superimposed lowest-energy structures of NSP7 $\alpha$ in aqueous solution. (B) The cartoon representation of NSP7 $\alpha$ structure in solution. (C) The surface representation of the solution structure of NSP7 $\alpha$. Negatively charged patches are colored in red, and positively charged regions are in blue. 


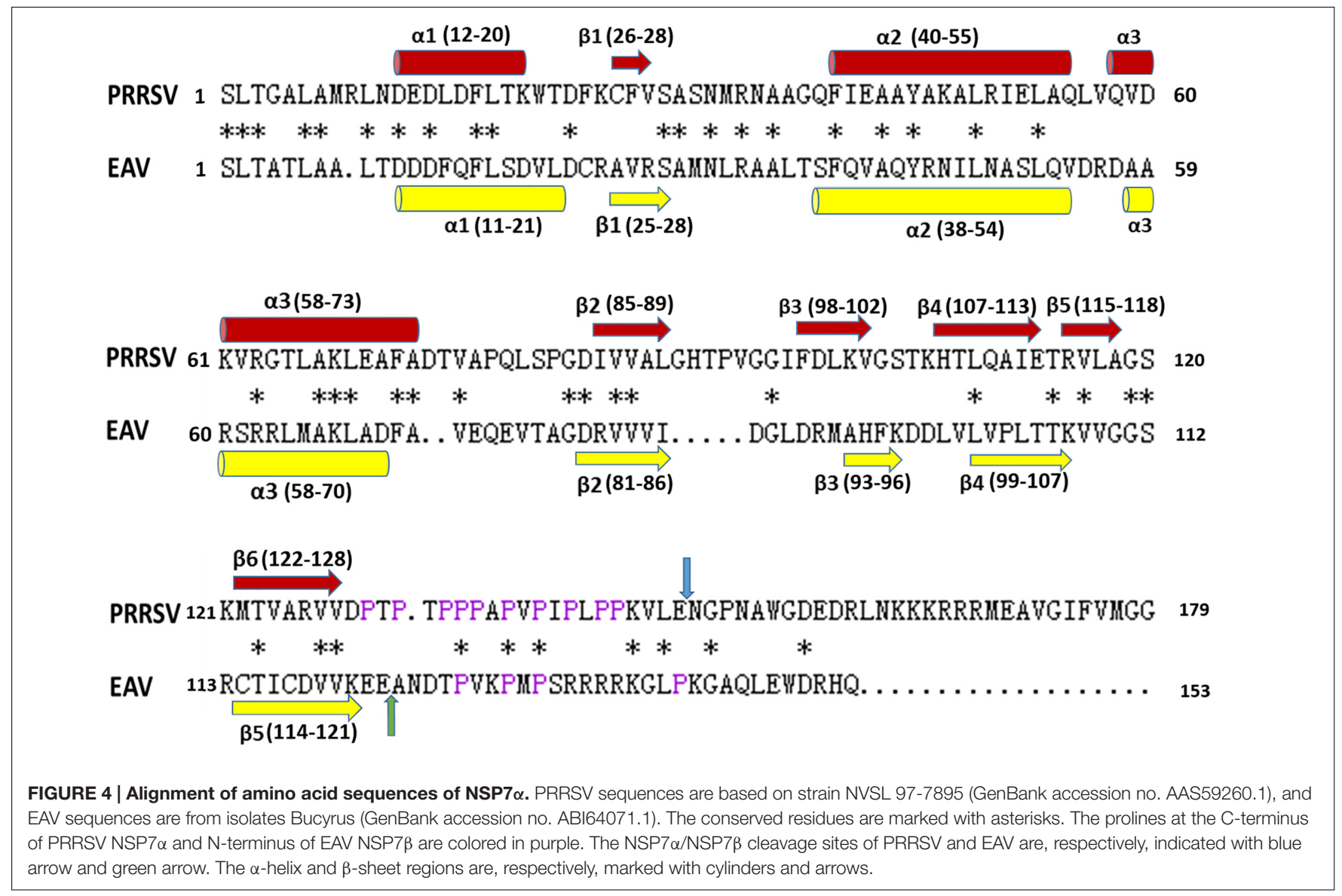
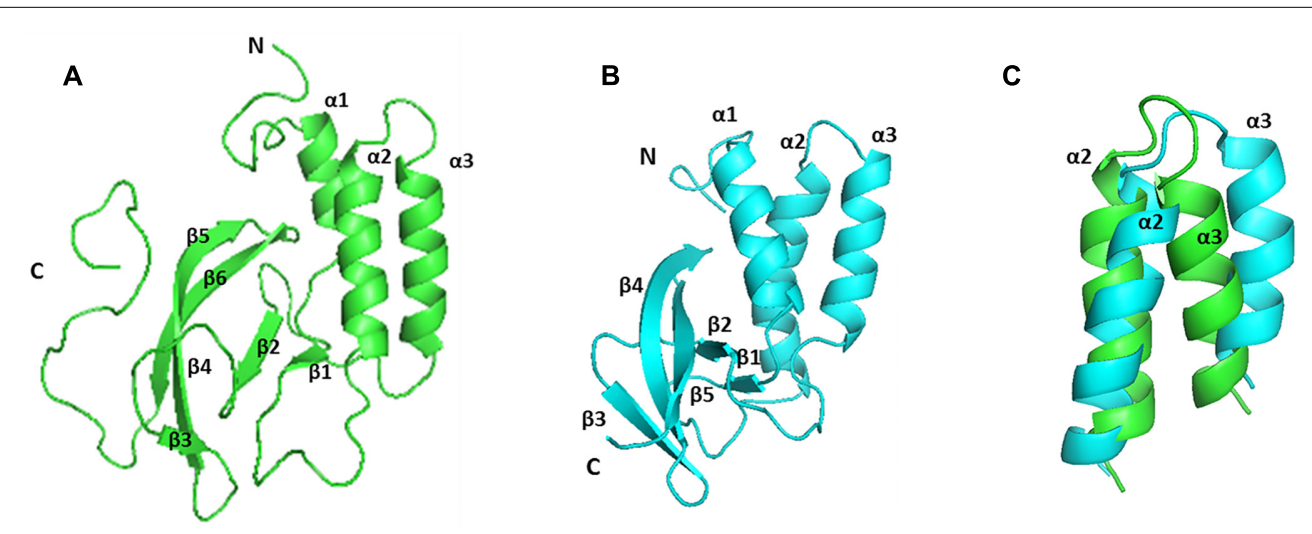

FIGURE 5 | Comparison of the solution structure of PRRSV and EAV NSP7 $\alpha$ proteins. (A) The structure of PRRSV NSP7 $\alpha$ (PDB accession number: 5165$).$ (B) The structure of EAV NSP7 $\alpha$ (PDB accession number: 2L8K). (C) Overlay of the region from helices $\alpha 2$ to $\alpha 3$. PRRSV NSP7 $\alpha$ is colored in green and EAV NSP7 $\alpha$ is in blue.

less identity with the N-terminus of PRRSV NSP7 $\beta$. Therefore, this discrepancy could be attributed to the position change of the cleavage site between NSP7 $\alpha$ and NSP7 $\beta$. It is unfortunate that the structure of the C-terminal PRR was not solved, as prolines lack the amide protons necessary for the standard triple resonance experiment and the backbone of this region was not able to be assigned.
The solution structure of EAV NSP7 $\alpha$ (PDB accession number: 2L8K) have previously been reported (Manolaridis et al., 2011). PRRSV NSP7 $\alpha$ possesses similar secondary structures as EAV NSP7 $\alpha$ (Figures 4, 5A,B). Both of them have three $\alpha$-helices gathered together in the $\mathrm{N}$-terminal half, and all the $\beta$-sheets of the two proteins are located at one side of the $\alpha$-helices. Strand $\beta 4$ and strand $\beta 5$ of PRRSV NSP7 $\alpha$ locate at a similar 


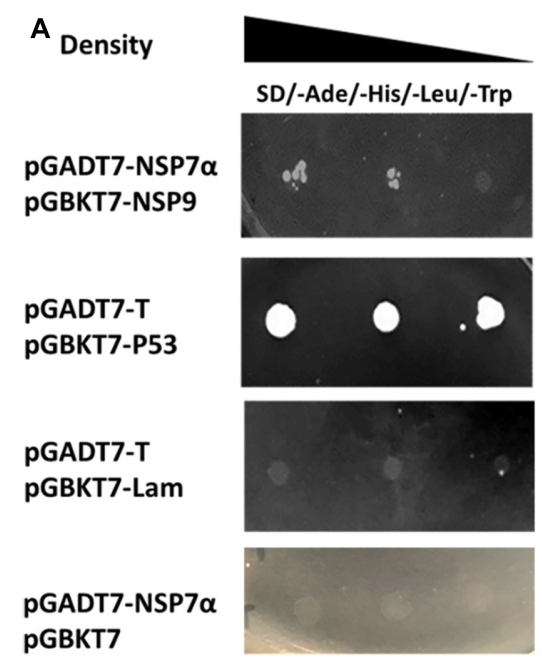

B
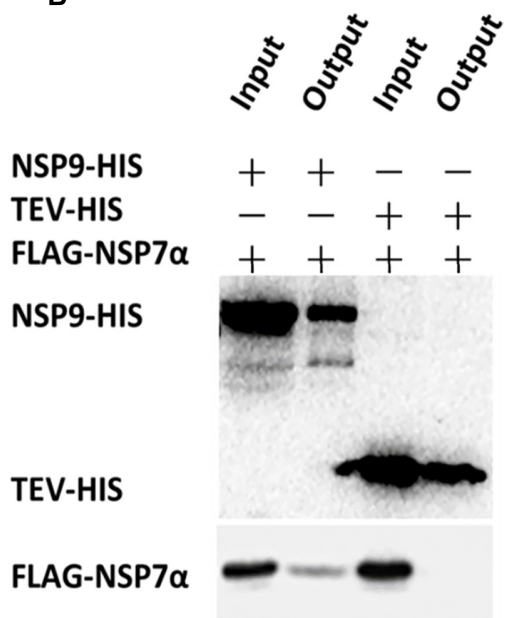

FIGURE 6 | Identification of the interaction of NSP7 $\alpha$ with NSP9. (A) Yeast two-hybrid assays. The yeast cells were co-transformed with pGADT7-nsp7 $\alpha$ and pGBKT7-nsp9 constructs, subjected to 10-fold serial dilutions and plated on SD/-Ade/-His/-Leu/-Trp medium. The cells co-transformed with pGBK7-P53 and pGADT7-T were used as positive interaction controls, and the group with pGBKT7-Lam and pGADT7-T was used as a negative control. (B) Pull-down assays. FLAG-tagged NSP7 $\alpha$ and HIS-tagged NSP9 were expressed in E. coli, mixed together, and the protein mixture was purified with nickel magnetic beads. HIS-tagged and FLAG-tagged proteins pulled down by the beads were, respectively, analyzed by Western blot with antibodies against HIS-tag and FLAG-tag.

position with an equivalent length to the strand $\beta 4$ of EAV NSP7 $\alpha$. However, overlay of the 3-D structure of the two proteins showed that the two structures were not well superimposed, and the best superimposed region located from helices $\alpha 2$ to helices $\alpha 3$ (Figure 5C).

\section{Interaction of NSP7 $\alpha$ with NSP9}

Previous studies have reported that arterivirus NSP7 $\alpha$ is essential for the viral replication and it plays an important role in viral RNA synthesis (Manolaridis et al., 2011; Zhang et al., 2013). It remains unknown whether the protein is involved in the replication and transcription complex (RTC) formation by interacting with other NSPs. To investigate the interaction of PRRSV NSP7 $\alpha$ with other NSPs, yeast two-hybrid assays were carried out, and it was found that NSP7 $\alpha$ could interact with NSP9 (Figure 6A), the viral RNA-dependent RNA polymerase. To validate the NSP7 $\alpha$-NSP9 interaction, FLAG-tagged NSP7 $\alpha$ and HIS-tagged NSP9 were expressed in E. coli and used for pulldown assay. The data confirmed that NSP7 $\alpha$ could bind to NSP9 in vitro (Figure 6B).

To investigate the interaction of NSP7 $\alpha$ with NSP9 in more details, the 3-D structure of NSP9 was predicted (Liu et al., 2016), and used for the docking prediction of NSP7 $\alpha-$ NSP9 interaction by using PRISM2.0 (Tuncbag et al., 2011). In the predicted interaction model with the lowest energy $(-27.86 \mathrm{kcal} / \mathrm{mol})$ among the outputs, three amino acids (K61, L69, and F72) in helix $\alpha 3$ of NSP7 $\alpha$ were predicted to be involved in the recognition of NSP9 (Figure 7). Pull-down assay using the NSP7 $\alpha$ mutants containing single substitutions at the three sites showed that mutations at L69 and F72 abolished the binding ability of NSP7 $\alpha$ to NSP9, but the K61A mutant could interact with NSP9 as well as WT NSP7 $\alpha$ (Figure 8). It is worth to note that L69 and F72 were conserved between PRRSV and EAV NSP7 $\alpha$, while K61 in PRRSV NSP7 $\alpha$ was replaced by another basic amino acid $\mathrm{R}$ in EAV NSP7 $\alpha$ (Figure 4). This result confirmed that helix $\alpha 3$ of NSP7 $\alpha$ was a critical region involved in the NSP7 $\alpha-\mathrm{NSP} 9$ interaction, and it also indicated that the two conserved amino acids L69 and F72 were essential for the protein to maintain its ability binding to NSP9.

\section{DISCUSSION AND CONCLUSION}

NSP7 $\alpha$ is one of the most conservative NSPs of PRRSV and is an essential factor for the rescue of virus by reverse genetics (Zhang et al., 2013), but its structure and specific function in virus biology still remains unknown. To elucidate the structure and function of NSP7 $\alpha$, we analyzed its solution structure, the expression of the protein in PRRSV infected cells, and its interaction with NSP9 in this study.

Previous studies found that NSP7 of arterivirus EAV could be internally cleaved by NSP4, the viral protease, yielding small amounts of NSP7 $\alpha$ and NSP7 $\beta$. In infected cell lysates, NSP7 and its cleaved product NSP7 $\alpha$ were detected by Western blot. Cleaved NSP7 $\beta$ was not detected, but mutagenesis analysis demonstrated that the internal processing of NSP7 was critical for virus reproduction (van Aken et al., 2006). A recent study on PRRSV NSP7 showed that only NSP7 $\alpha$ was detectable in the infected cells, and neither NSP7 nor NSP7 $\beta$ were detected by Western blot and radioimmunoprecipitation using monoclonal antibodies against NSP7 $\alpha$ and NSP7 $\beta$ (Li et al., 2012).

Here, by Western blot using polyclonal antibodies against NSP7 $\alpha$ and NSP7 $\beta$, we found that cleaved NSP7 $\alpha$ could be detected at 16 hpi, but NSP7 and NSP7 $\beta$ were still undetectable in the cell lysates infected by PRRSV. Recombinant NSP7 $\beta$ had 


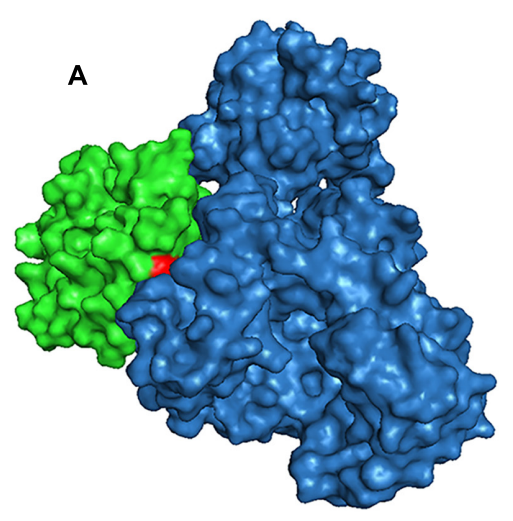

C

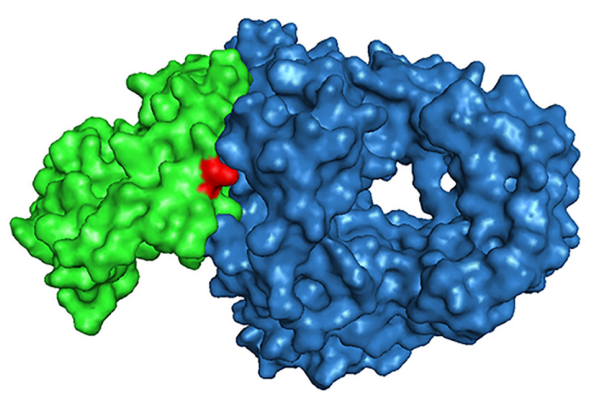

B
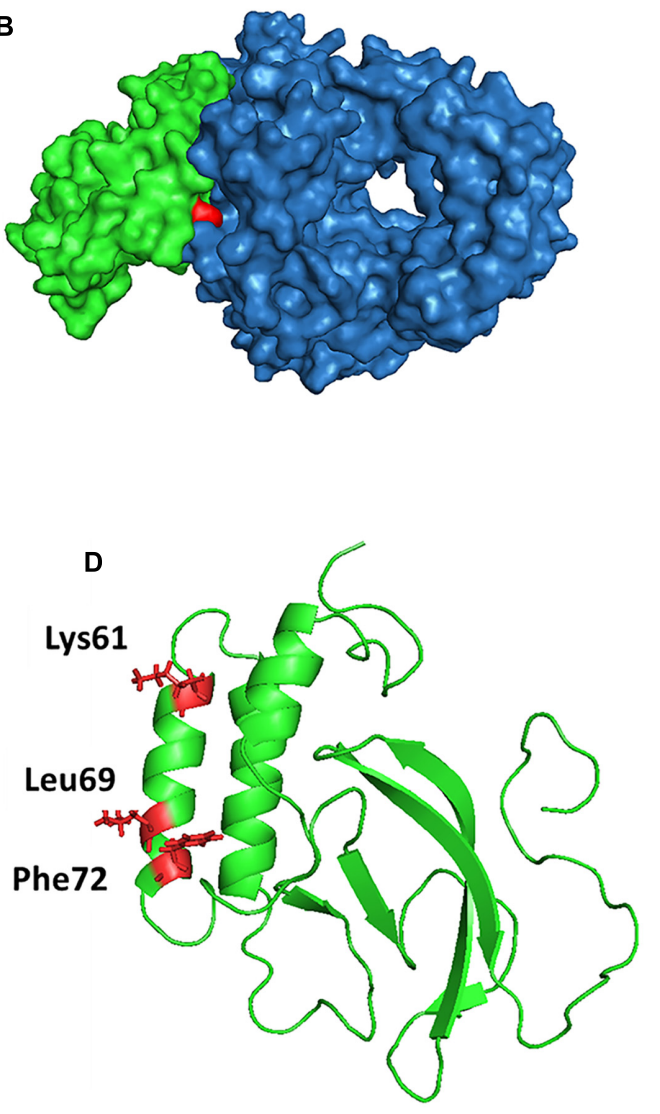

FIGURE 7 | Prediction model of NSP7 $\alpha-$ NSP9 interaction (with evaluated energy of $\mathbf{- 2 7 . 8 6} \mathbf{k c a l} / \mathbf{m o l}$ ). The surface features of the NSP7 $\alpha-N S P 9$ interaction model are shown in (A-C). NSP7 $\alpha$ is colored in green, NSP9 is in blue, and Lys61 (A), Leu69 (B), and Phe72 (C) are highlighted in red. (D) The three amino acid residues are highlighted in red in the cartoon representation of NSP7 $\alpha$ structure.

been observed to be sensitive to degradation (Li et al., 2012). We speculated that the cleaved NSP7 $\beta$ could be rapidly degraded in virus infected cells, which resulted in the failures in the detection of the protein. For NSP7, however, we found in our studies that it could be abundantly expressed in E. coli and the purified protein was also quite stable (data not shown). Taken together with the fact that both antisera against NSP7 $\alpha$ and NSP7 $\beta$ detected no NSP7 but some larger processing intermediates such as NSP3-8 and NSP5-7, our results indicated that PRRSV NSP7 might only exist as a short-lived intermediate if it was present. NSP7 $\alpha$ was found stably existing as a cleavage product in PRRSV infected cells after $16 \mathrm{hpi}$, suggesting that it may play an important role in the virus infection. Whether the long-lived intermediates such as NSP3-8 and NSP5-7 act as functional proteins remains to be investigated.

It is noteworthy that PRRSV NSP7 $\alpha$ contains a predominant $\mathrm{PRR}$ at the $\mathrm{C}$-terminus. Its homologous region containing less proline residues presents at the N-terminus of EAV NSP7 $\beta$, probably due to the cleavage site shift between NSP7 $\alpha$ and NSP7 $\beta$. PRRs widely exist in proteins and often have important functions. The proline-rich peptides are found to be especially involved in protein-protein interactions, such as the recognition

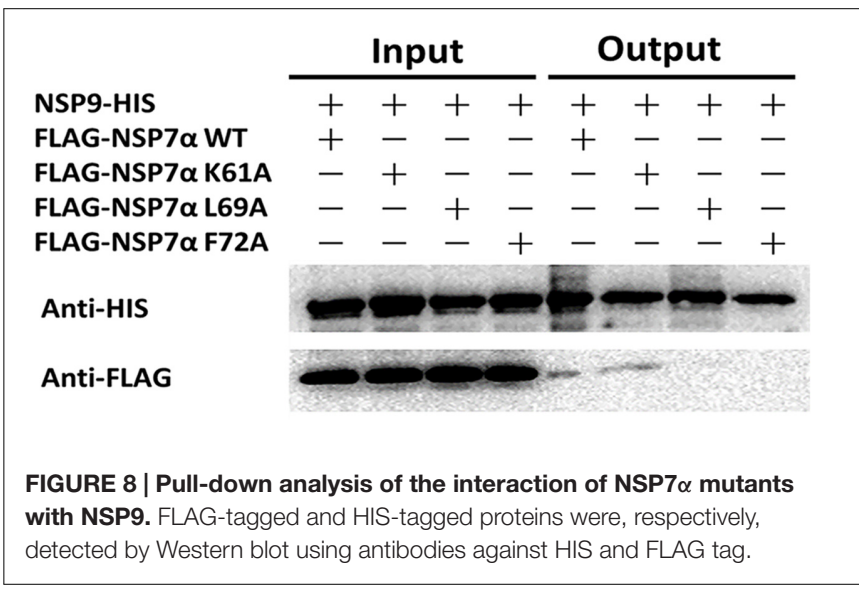

of regulatory proteins with $\mathrm{SH} 3$ domains in signaling pathways. It has been found that the PRR in proteins with repetitive $(X P)_{n}$ sequences can function as a stiff 'sticky arm, binding rapidly and reversibly to other proteins (Williamson, 1994). The C-terminal PRR of PRRSV NSP7 $\alpha$ has a sequence pattern of $(X P)_{8}$ (Figure 4). 
It is a pity that we were unable to resolve the structure of this region due to the restriction of the NMR method we used in this study. Further studies are needed to understand whether this extra motif endows PRRSV NSP7 $\alpha$ with any extra function than EAV NSP7 $\alpha$.

Structure analysis and comparison show that PRRSV NSP7 $\alpha$ (PDB accession No. 5I65) and EAV NSP7 $\alpha$ (PDB accession No. 2L8K) possess similar secondary structure elements (Figure 5). Both of them have three $\alpha$-helices gathered together, and $\beta$-sheets of the two proteins are all located at one side of the $\alpha$-helices. Helix $\alpha 2$ to helix $\alpha 3$ is the best superimposed region of two proteins (Figure 5C).

By yeast two hybrid and pull-down assays, we found that PRRSV NSP7 $\alpha$ could interact with its viral RdRp. Site-directed mutagenesis analysis showed that NSP7 $\alpha$ single mutants L69A and F72A lost the binding ability to NSP9. Interestingly, a previous study showed that a single mutation at F72 resulted in the failure in virus recovery by reverse genetics (Zhang et al., 2013). F72 is a conserved amino acid residue in NSP7 $\alpha$ of PRRSV and EAV, indicating that it is probably a crucial residue for the protein to maintain its functional structure. Previous studies by reverse genetics have demonstrated that arterivirus NSP7 $\alpha$ plays an important role in viral RNA synthesis (Manolaridis et al., 2011; Zhang et al., 2013). NSP9 acts as the viral RNA-dependent RNA polymerase and is a key component of the viral RTC (Knoops et al., 2012). The interaction of NSP7 $\alpha$ with NSP9 imply that NSP7 $\alpha$ might play a role in RTC and/or assist NSP9 to carry out its function in PRRSV RNA synthesis. Further work is needed to investigate whether

\section{REFERENCES}

Allende, R., Lewis, T. L., Lu, Z., Rock, D. L., Kutish, G. F., Ali, A., et al. (1999). North American and European porcine reproductive and respiratory syndrome viruses differ in non-structural protein coding regions. J. Gen. Virol. 80, 307-315. doi: 10.1099/0022-1317-80-2-307

Brown, E., Lawson, S., Welbon, C., Gnanandarajah, J., Li, J., Murtaugh, M. P., et al. (2009). Antibody response to porcine reproductive and respiratory syndrome virus (PRRSV) nonstructural proteins and implications for diagnostic detection and differentiation of PRRSV types I and II. Clin. Vaccine Immunol. 16, 628-635. doi: 10.1128/CVI.00483-08

Brünger, A. T., Adams, P. D., Clore, G. M., DeLano, W. L., Gros, P., GrosseKunstlevea, R. W., et al. (1998). Crystallography \& NMR system: a new software suite for macromolecular structure determination. Acta. Cryst. 54, 905-921.

Chen, J., Xu, X., Tao, H., Wang, Y., and Chen, H. (2016). Chemical shift assignments of nsp7alpha from porcine reproductive and respiratory syndrome virus. Biomol. NMR Assign. 10, 391-394. doi: 10.1007/s12104-016-9706-7

Delaglio, F., Grzesiek, S., Vuister, G. W., Zhu, G., Pfeifer, J., and Bax, A. (1995). NMRPipe: a multidimensional spectral processing system based on UNIX pipes. J. Biomol. NMR 305, 277-293.

Dunowska, M., Biggs, P. J., Zheng, T., and Perrott, M. R. (2012). Identification of a novel nidovirus associated with a neurological disease of the Australian brushtail possum (Trichosurus vulpecula). Vet. Microbiol. 156, 418-424. doi: 10.1016/j.vetmic.2011.11.013

Fang, Y., and Snijder, E. J. (2010). The PRRSV replicase: exploring the multifunctionality of an intriguing set of nonstructural proteins. Virus Res. 154, 61-76. doi: 10.1016/j.virusres.2010.07.030

Forsberg, R. (2005). Divergence time of porcine reproductive and respiratory syndrome virus subtypes. Mol. Biol. Evol. 22, 2131-2134. doi: 10.1093/molber/msi208 and how the NSP7 $\alpha$-NSP9 interaction is involved in the virus replication.

To conclude, in this study, we investigated the expression of NSP7 $\alpha$ and NSP7 $\beta$ in PRRSV-infected cells, resolved the structure of NSP7 $\alpha$ in aqueous solution, and identified the interaction of NSP7 $\alpha$ with the viral RNA dependent RNA polymerase NSP9. These data provided us some new insights into the molecular mechanism of PRRSV infectious cycle.

\section{AUTHOR CONTRIBUTIONS}

HC, XX, and JC designed the research; JC, XX, HT, and YW determined the solution structure of NSP7 $\alpha$; YL, HN, and MT demonstrated the interaction of NSP7 $\alpha$ with NSP9; JC performed the rest of the experiments; JC, HC, and XX contributed to the writing of manuscript.

\section{FUNDING}

This research was supported by the Fundamental Research Funds for the Central Universities (QN2011065).

\section{ACKNOWLEDGMENTS}

The authors thank Beijing NMR center for the expert technical assistance and Pengfei Ding at Peking University for his help in analysis of spectra.

Keller, R. (2004). Optimizing the Process of Nuclear Magnetic Resonance Spectrum Analysis and Computer Aided Resonance Assignment. Doctoral thesis, Swiss Federal Institute of Technology, Zurich.

Knoops, K., Barcena, M., Limpens, R. W., Koster, A. J., Mommaas, A. M., and Snijder, E. J. (2012). Ultrastructural characterization of arterivirus replication structures: reshaping the endoplasmic reticulum to accommodate viral RNA synthesis. J. Virol. 86, 2474-2487. doi: 10.1128/JVI. 06677-11

Kwon, B., Ansari, I. H., Pattnaik, A. K., and Osorio, F. A. (2008). Identification of virulence determinants of porcine reproductive and respiratory syndrome virus through construction of chimeric clones. Virology 380, 371-378. doi: 10.1016/j.virol.2008.07.030

Laskowski, R. A., Rullmann, J. A. C., MacArthur, M. W., Kaptein, R., and Thornton, J. M. (1996). AQUA and PROCHECK-NMR: programs for checking the quality of protein structures solved by NMR. J. Biomol. NMR 8, 477-486.

Li, Y., Tas, A., Snijder, E. J., and Fang, Y. (2012). Identification of porcine reproductive and respiratory syndrome virus ORF1a-encoded non-structural proteins in virus-infected cells. J. Gen. Virol. 93(Pt 4), 829-839. doi: 10.1099/ vir.0.039289-0

Li, Y., Tas, A., Sun, Z., Snijder, E. J., and Fang, Y. (2015). Proteolytic processing of the porcine reproductive and respiratory syndrome virus replicase. Virus Res. 202, 48-59. doi: 10.1016/j.virusres.2014.12.027

Liu, L., Tian, J., Nan, H., Tian, M., Li, Y., Xu, X., et al. (2016). Porcine reproductive and respiratory syndrome virus nucleocapsid protein interacts with Nsp9 and cellular DHX9 to regulate viral RNA synthesis. J. Virol. 90, 5384-5398. doi: 10.1128/JVI.03216-15

Manolaridis, I., Gaudin, C., Posthuma, C. C., Zevenhoven-Dobbe, J. C., Imbert, I., Canard, B., et al. (2011). Structure and genetic analysis of the arterivirus nonstructural protein 7 $\alpha$. J. Virol. 85, 7449-7453. doi: 10.1128/JVI.00255-11 
Nelsen, C. J., Murtaugh, M. P., and Faaberg, K. S. (1999). Porcine reproductive and respiratory syndrome virus comparison divergent evolution on two continents. J. Virol. 73, 270-280.

Plagemann, P. G. W., and Moennig, V. (1992). Lactate dehydrogenaseelevating virus, equine arteritis virus, and simian hemorrhagic fever virus: a new group of positive-strand RNA viruses. Adv. Virus. Res. 41, 99-192.

Shen, Y., Delaglio, F., Cornilescu, G., and Bax, A. (2009). TALOS+: a hybrid method for predicting protein backbone torsion angles from NMR chemical shifts. J. Biomol. NMR 44, 213-223. doi: 10.1007/s10858-0099333-z

Snijder, E. J., and Meulenbdrg, J. J. M. (1998). The molecular biology of arteriviruses. J. Gen. Virol. 79, 961-979.

Tuncbag, N., Gursoy, A., Nussinov, R., and Keskin, O. (2011). Predicting proteinprotein interactions on a proteome scale by matching evolutionary and structural similarities at interfaces using PRISM. Nat. Protoc. 6, 1341-1354. doi: 10.1038/nprot.2011.367

van Aken, D., Zevenhoven-Dobbe, J., Gorbalenya, A. E., and Snijder, E. J. (2006). Proteolytic maturation of replicase polyprotein ppla by the nsp4 main proteinase is essential for equine arteritis virus replication and includes internal cleavage of nsp7. J. Gen. Virol. 87(Pt 12), 3473-3482.
Williamson, M. P. (1994). The structure and function of proline-rich regions in proteins. Biochem. J. 297, 249-260.

Zhang, M., Cao, Z., Xie, J., Zhu, W., Zhou, P., Gu, H., et al. (2013). Mutagenesis analysis of porcine reproductive and respiratory syndrome virus nonstructural protein 7. Virus Genes 47, 467-477. doi: 10.1007/s11262-0130957-4

Conflict of Interest Statement: The authors declare that the research was conducted in the absence of any commercial or financial relationships that could be construed as a potential conflict of interest.

The reviewer TO and handling Editor declared their shared affiliation, and the handling Editor states that the process nevertheless met the standards of a fair and objective review.

Copyright (c) 2017 Chen, Xu, Tao, Li, Nan, Wang, Tian and Chen. This is an openaccess article distributed under the terms of the Creative Commons Attribution License (CC BY). The use, distribution or reproduction in other forums is permitted, provided the original author(s) or licensor are credited and that the original publication in this journal is cited, in accordance with accepted academic practice. No use, distribution or reproduction is permitted which does not comply with these terms. 